- it has been difficult, however ....

...engineer $40 \mathrm{hrs} /$ week (=50 per cent of time) + mother (more an art than a science) ....

... worked half time for 12 years while children were small ....

And, from someone identifying herself as a housewife/programmer analyst, "my husband's job and the two children have taken precedence."

\title{
Acknowledgments
}

Variable-star research at the Maria Mitchell Observatory has received funding from the National Science Foundation, currently under AST86-19885, which includes an allotment from the program Research Experiences for Undergraduates. Since 1979, one of the students each summer receives support from the Dorrit Hoffleit Assistantship Fund of the Maria Mitchell Association. The use of remote photoelectric observing was funded by a Theodore Dunham, Jr., grant for research in astronomy.

\section{THE EDINBURGH ASTRONOMY TEACHING AND EDUCATIONAL PACKAGES}

\section{M.T. Brück and S.B. Tritton ${ }^{1}$}

Department of Astronomy, University of Edinburgh

${ }^{1}$ U.K. Schmidt Telescope Unit, Royal Observatory Edinburgh, Blackford Hill,

Edinburgh EH9 3HJ, Scotland

\section{Introduction}

In this paper we describe our experiences with film copies of original astronomical photographs taken with the 1.2-meter U.K. Schmidt Telescope (UKST) in Australia, and used as teaching material in the Department of Astronomy of the University of Edinburgh. Two packages are intended for undergraduate use; the Education Packages are designed as visual aids for colleges, schools, and amateur groups.

The original purpose of the telescope (which was commissioned in 1973) was to carry out a Southern Sky Survey to match the Northern Survey done by the Palomar 48" Schmidt Telescope. The telescope has a very wide field $-6.5 \times 6.5$ degrees, or equivalent to over a hundred and fifty full moons, and the photographs reach objects of 23rd magnitude: they record stars like the Sun to the very edges 
of the Galaxy and galaxies to a thousand million light years. Each photograph has an area of $356 \times 356 \mathrm{~mm}$ and records between 100,000 and 1 million stars and galaxies. The survey photographs are the deepest available maps of the sky and are indispensable tools for astronomers in searches for unusual objects, for investigation of the distribution of galaxies and many other tasks. The UKST is equipped with objective prisms that are capable of producing low dispersion spectra of stars and galaxies. A prism of very small angle is placed in front of the aperture of the telescope so that each individual image is drawn out into a tiny spectrum on the photograph. The dispersion is very small, only a few millimeters from the red to the ultraviolet, but the dominant features in the spectra are recognizable and have many applications.

The films that we use in our Teaching Packages are, like the original photographs, negatives, with the stars as black images on a transparent sky background. The original photographs are taken on glass; the copies are contact reproductions on film (as is the Southern Sky Survey) so that every detail on the original is retained. The use of these films for teaching purposes has many advantages. On the purely practical level they are relatively cheap; they are also unbreakable and, if kept enclosed in their transparent envelopes and treated with common-sense care, have an indefinite lifetime. A major attraction is that they constitute the highest quality modern observational material such as is currently used for advanced astronomical research.

Much of the research carried out with Schmidt photographs involves measuring dimensions of features, counting numbers of objects, and classifying objects. Our exercises also involve measuring, counting, and classifying. Some exercises are elementary and can be carried out in about one hour by first-year students, many of whom are non-physicists. For these, the only equipment required is a millimeter ruler, a light box, and a hand magnifier (preferably fitted with a graticule). For some of the other exercises a light table with a high-power microscope is recommended and a simple photometer allows one or two additional exercises to be done. At Edinburgh, we also use the films for more advanced projects that are in the nature of research projects occupying up to thirty hours for final year honors students of astrophysics: students can carry out genuine projects that yield answers to real problems and in which they encounter real difficulties.

The photographs need to be accompanied by other information and by some level of theoretical knowledge. We have included the relevant additional data with each exercise to make it self-contained. The exercises have become part of the practical course in astrophysics in the University of Edinburgh and have been found very successful not only for the experience of carrying them out but also for the results.

In our Packages, we have chosen sets of films that include examples of typical objects of astrophysical interest ranging from the solar system to distant galaxies. Worked solutions for four of the simple exercises and a summary of one more advanced exercise are given in the Appendices at the end of this paper. 


\section{The Solar System}

Our first example (Appendix 1) is the simplest, a study of images of asteroids on photographs of fields in the plane of the ecliptic. During the one-hour exposure time, asteroids that are in motion relative to the background of fixed stars leave trails rather than dots on the photograph. The actual calculation in the exercise is in principle simple: to deduce, from the length of the trail, the distance of the asteroid from the Sun assuming circular orbits and Kepler's third law. The practical matter of converting trail lengths to circular angular measure and of realizing that the final answer has to be obtained by trial, makes this an excellent puzzle, which takes about an hour to solve.

The exercises on comets (Appendix 2) are at a similar level of difficulty. The student is asked to demonstrate that the comet's tail points away from the Sun and to calculate the unforeshortened length of the tail, given appropriate information about the comet's path. The two films of Comet Halley taken on successive days show moving knots of material in the comet's ionic tail. The speed of the knots, which are being swept along in the direction of the solar wind, can be calculated.

\section{Galactic Objects}

Moving outwards among the stars, there are examples of various typical constituents of our Galaxy - star clusters, dark dust clouds, reflection nebulae, and a supernova remnant. The Vela supernova remnant is a luminous shell of ionized gas that outlines the boundary between the material bursting outwards from the explosion of a star in the final stages of its evolution, and the interstellar medium through which it moves. By chance the whole of this shell fits into one Schmidt photograph. The distance of the remnant is known from radio observations. Radio observations have also discovered the pulsar that is the remains of the exploded star, located about one degree away from the center of the shell, having recoiled at the explosion from its original position. The practical part of the exercise, in which the linear dimensions of the shell and the linear displacement of the pulsar are calculated, can be done within one laboratory session. The exercise also introduces the student to the theory of shocks in the interstellar medium.

So far the exercises involve only linear measurement. We now describe some that involve the counting of images and which are intended for longer projects. We have an example of a globular star cluster (Appendix 3). The question is how massive must a cluster be in order to withstand what is in effect the tidal force of the Galaxy. The mass is calculated indirectly by observing the apparent distribution of stars in its outer regions. The task set in the exercise is to count the numbers of stars per arbitrary unit of area radially outwards from the center of the cluster; at least several hundred images have to be counted. The exercise is a test of care and patience; it is incidentally also valuable practice in dealing with number statistics. An advanced exercise involves matching the distribution of stars in the cluster to a mathematical model.

We have also chosen a typical field in the galactic plane that very strikingly 
illustrates the enormous numbers of stars in the Milky Way and the presence of dark clouds of interstellar material. In fact, the apparent dearth of stars in some patches of sky is caused by loss of light from the stars behind, through scattering by small particles of dust. By counting the relative densities of stars inside and outside the area of a dark cloud, it is possible to calculate the fraction of light being removed by the dust. From the known properties of scattering grains, the total number of grains in the line of sight may be calculated and an estimate made of the mass of the cloud. The actual star-counting part of this exercise is not difficult in principle, but the result is exciting and the student is introduced to the theory of the scattering of light.

Two films taken with the objective prism are provided (Appendix 4); one is of a Milky Way field and the other of a high galactic latitude field. At these dispersions only the dominant features in the spectra can be distinguished. Spectral classification into the main classes is an obvious exercise that is particularly instructive if it is performed on contrasting regions in the sky. The differences are reflected in the spectral types of the stars, and it is very satisfying for a student to discover this.

\section{Extragalactic - The Magellanic Clouds}

In the extragalactic sphere there are many possibilities for student exercises to be found on photographs of the Magellanic Clouds. On deep photographs they extend over a large area of sky and appear on several Schmidt photographs of which a few have been selected for our Packages. One exercise is a survey of star clusters in the Large Cloud. The Large Magellanic Cloud has an irregular spiral structure and is fortuitously oriented in such a way in space that from the point of view of the observer on Earth its disk is seen almost face-on. Several hundred clusters, easily recognizable under low magnification, are identified. Their average number density plotted as a function of radial distance from the center gives the distribution of mass within the disk. The distribution turns out to be exponential, in accordance with standard models of galactic disks.

An important use of objective prism spectra is to search for objects with unusual characteristics, in particular objects with emission-line spectra that show up conspicuously on photographs by contrast with the normal absorption spectra of the vast majority of stars and galaxies. Emission lines are found in very young stars with hot gaseous envelopes and in planetary nebulae. The spectra of these types are distinctive and a student soon learns to tell the difference. An objective prism film of part of the LMC where there are large numbers of emission line objects of all kinds has been chosen for this exercise.

\section{Extragalactic - Clusters of Galaxies and Quasars}

One of the most useful films in the Package is a photograph of the Virgo cluster of galaxies. The most obvious use of the photograph is for the classification of galaxies according to the "tuning-fork" scheme that distinguishes elliptical from spiral galaxies and further subdivisions. The photograph shows such fine detail that 
an exercise can also include measuring the diameters of galaxies, estimating their linear dimensions and observing the number of spiral arms in the giant ones. The same photograph shows many very distant background galaxies whose distances may be inferred from their apparent diameters.

Another example is Abell 1060 (Appendix 5), a cluster of galaxies rather similar to the Virgo cluster but at a considerably greater distance. This advanced exercise leads to quite a sophisticated analysis of the internal dynamics of the system when the spatial separation of the galaxies as deduced from counts is combined with motions of individual galaxies, which are known from spectroscopic Doppler shift observations. The final result is a good estimate of the total mass of the cluster. There is a considerable amount of recording and computation and, if carefully done, the result obtained bears comparison with the research investigator's. It is especially instructive to compare the mass calculated by this method with that expected from the total sum of individual galaxies. The student, taking all the uncertainties into account, finds himself debating as to whether there is or is not evidence for "missing" or invisible mass in the cluster.

An exciting exercise is to search for quasars that are identified from their peculiar emission line spectra. There are thousands as yet undiscovered on UKST objective prism photographs. A student, with no more equipment than a hand lens, can expect to find 30 or 40 on each of the films which we have included in our Package; with rather more labor he can measure their redshifts and, adopting a suitable model for the expanding Universe, estimate their distances.

\section{Educational Packages}

These packages have been made up from film copies originally made for the ESO/SERC Southern Sky Survey but which failed the very stringent quality control criteria. Each package contains films of different areas of the sky and contains examples of most types of astronomical objects. Care has been taken to include films in each package which show the high density star fields of the Milky Way and also areas of the sky rich in galaxies. Comets, asteroids, star clusters, planetary nebulae, dust clouds, reflection and emission nebulae, and spiral and elliptical galaxies are all represented. Each film is accompanied by a sheet listing some of the interesting objects which can be seen. No attempt has been made to provide exercises for these films which are therefore mainly useful as visual aids.

\section{Details of Packages}

1. Edinburgh Astronomy Teaching Package for Undergraduates (M.T. Brück 1983, revised and expanded 1988) ( $£ 100)$ : Ten film copies together with a set of notes suggesting a variety of exercises. The notes are intended for teachers who can then choose the most suitable exercises for their particular class. General solutions and references, rather than complete detailed workings, are provided for numerical problems. The exercises are on Asteroids, Comets, Globular Clusters, the Galactic Plane, the Vela SNR, the LMC, and Clusters of Galaxies. 
2. Edinburgh Astronomy Educational Packages for Schools ( $£ 50$ per set): These packages are mainly designed as visual aids. Each package contains 10 films of different areas of the sky and each film is accompanied by a brief sheet describing the most interesting objects that can be seen. Five separate packs are available.

3. Edinburgh Astronomy Spectroscopic Teaching Package (M.T. Brück and S.B. Tritton, 1988) ( $£ 100$ ): Eight film copies ( 5 from plates taken with the objective prism and 3 from matching direct plates) and a set of notes suggesting exercises as in Package 1. The exercises include spectral typing, searching for emission line stars, and searching for quasars. The package also contains the Atlas of UKST objective prism spectra (Savage et al., 1985).

\section{Acknowledgments}

The authors thank Mrs. E. Gibson for her patient typing and the U.K. Schmidt Telescope Unit and Photolabs, Royal Observatory, Edinburgh.

\section{References}

M.T. Brück, 1986, "Photographic Teaching Packages in Astronomy," in Proceedings of the GIREP Conference 1986: Cosmos - an Educational Challenge. ESASP-253.

S.B. Tritton, 1986, "Educational Packages for Schools and Amateur Groups," in Proceedings of the GIREP Conference 1986: Cosmos - an Educational Challenge. ESA-SP-253.

A. Savage et al., 1985, "The UKST Objective Prisms." Royal Observatory, Edinburgh.

\section{Appendix 1: Asteroids or Minor Planets}

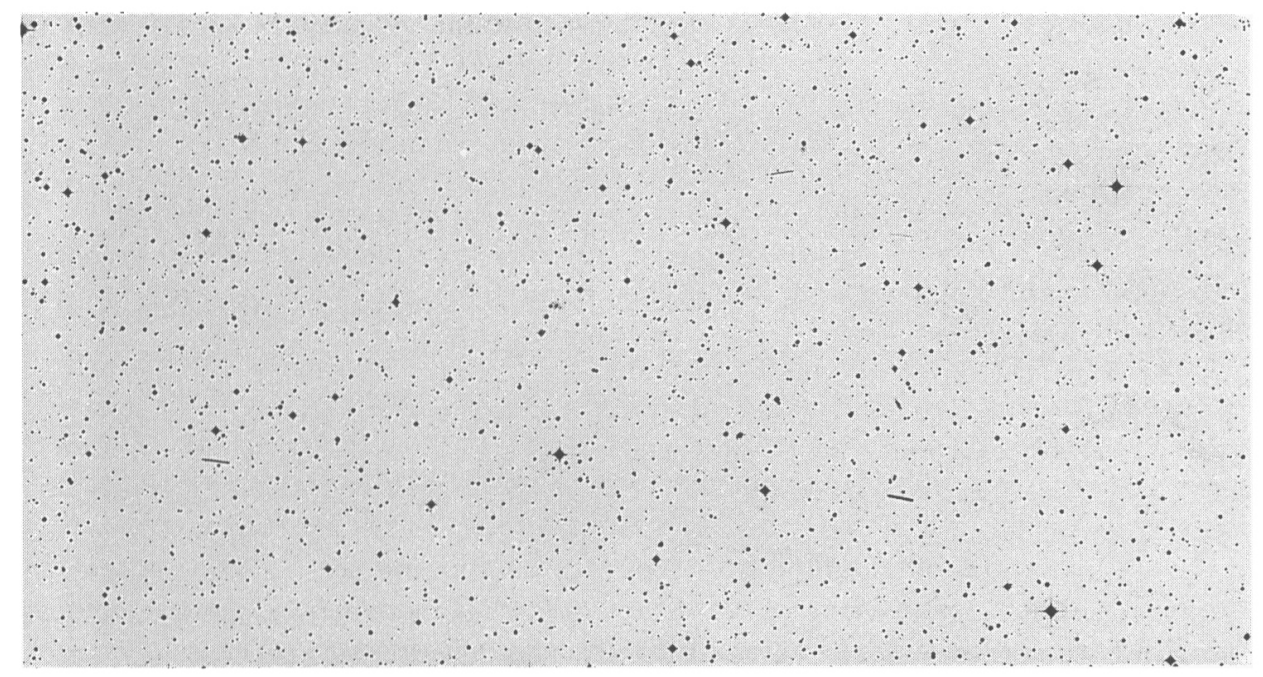

Plate 1: Several trailed images of asteroids in the ecliptic plane photographed at opposition. 
Exercise: Measure the length of the trails and calculate, assuming circular orbits, the distance of the asteroids from the Sun in Astronomical Units. The measurements are made with a graticule with $1 / 10 \mathrm{~mm}$ divisions, viewed on a light box with a x10 hand magnifier. Trail lengths are estimated to a 1/4 of a division.

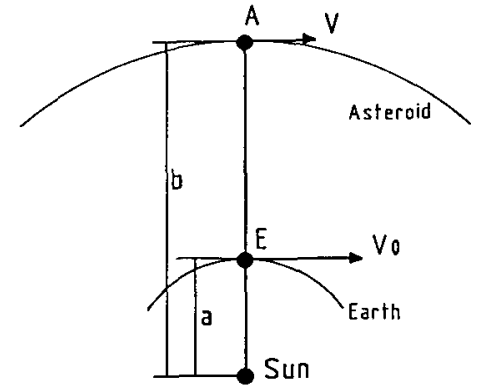

Figure 1a

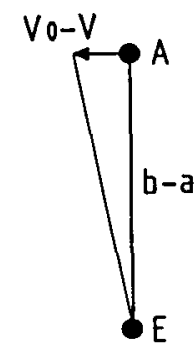

Figure $1 b$

Let $\quad \mathrm{a}=$ distance of the Earth from the Sun

$\mathrm{b}=$ distance of the asteroid from the Sun

$\mathrm{V}_{0}=$ Earth's orbital velocity

$\mathrm{V}=$ asteroid's orbital velocity

$\omega=$ angular motion of the asteroid as seen from Earth

$=\left(\mathrm{V}_{0}-\mathrm{V}\right) /(b-a)$ radians per second

By Kepler's third law, circular velocity is inversely proportional to the square root of the radius of the orbit, therefore

$$
\begin{aligned}
\mathrm{V}= & \mathrm{V}_{0}(a / \sqrt{b}), \text { which gives } \\
\omega= & \omega_{0} a(1-\sqrt{a / b}) /(b-a) \text { where } \omega_{0}=V_{0} / a, \text { the angular } \\
& \text { velocity of the Earth in its orbit around the Sun. }
\end{aligned}
$$

If $a$ and $b$ are in astronomical units, $a=1$.

$\omega_{0}$, the Earth's angular velocity, is 360 degrees per year $\left(3.16 \times 10^{7}\right.$ seconds $)$ or $360 \times 3600 / 3.16 \times 10^{7}$ seconds of arc per second (or minutes of arc per minute). Substituting the numbers and simplifying the algebra by factorizing $(b-1)$ into $(\sqrt{b}-1)(\sqrt{b}+1)$, one gets

$$
\begin{aligned}
\omega= & 4.10 \times 10^{-2} /(b+\sqrt{b}) \text { minutes of arc per minute for the } \\
& \text { rate at which the asteroid moves as seen from the Earth. }
\end{aligned}
$$

A typical trail on the film is $0.62 \mathrm{~mm}$ or 0.70 arcminutes (given the scale of the film of 1.12 arcminutes per $\mathrm{mm}$ ). The exposure time was 70 minutes; therefore the observed motion $\omega$ of the asteroid is 0.01 arcminutes per minute.

The problem is to find the value of $b$ that will make $\omega=0.01$, that means solving $b+\sqrt{b}=4.10$. It is quicker to find $b$ by the trial and error using a hand 
calculator than to solve the quadratic equation for $\sqrt{b}$. Try whole numbers first and then smaller steps: for $b=2, b+\sqrt{b}=3.41$; for $b=3, b=\sqrt{b}=4.73$; for $b=2.5$, $b=\sqrt{b}=4.08$.

Now estimate the accuracy of the result. The uncertainty lies in the measurement of the trail length (all other numbers used in the calculation are very accurate). The trail length was estimated to a quarter of a division, i.e., $0.025 \mathrm{~mm}$ in a length of 0.62 , or 1 part in 25 . The calculated motion of the asteroid and also the number $b+\sqrt{b}$ have therefore the same uncertainty of 1 part in 25 . If the calculation for $b$ is repeated using the upper and lower limits $(4.10 \pm 0.16)$ for $b+\sqrt{b}$, the limits for $b$ are found to be 2.4 and 2.6. Thus the result for the distance of the asteroid from the Sun, assuming circular orbit, may be given as $2.5 \pm 0.1$ Astronomical Units.

\section{Appendix 2: Halley's Comet}

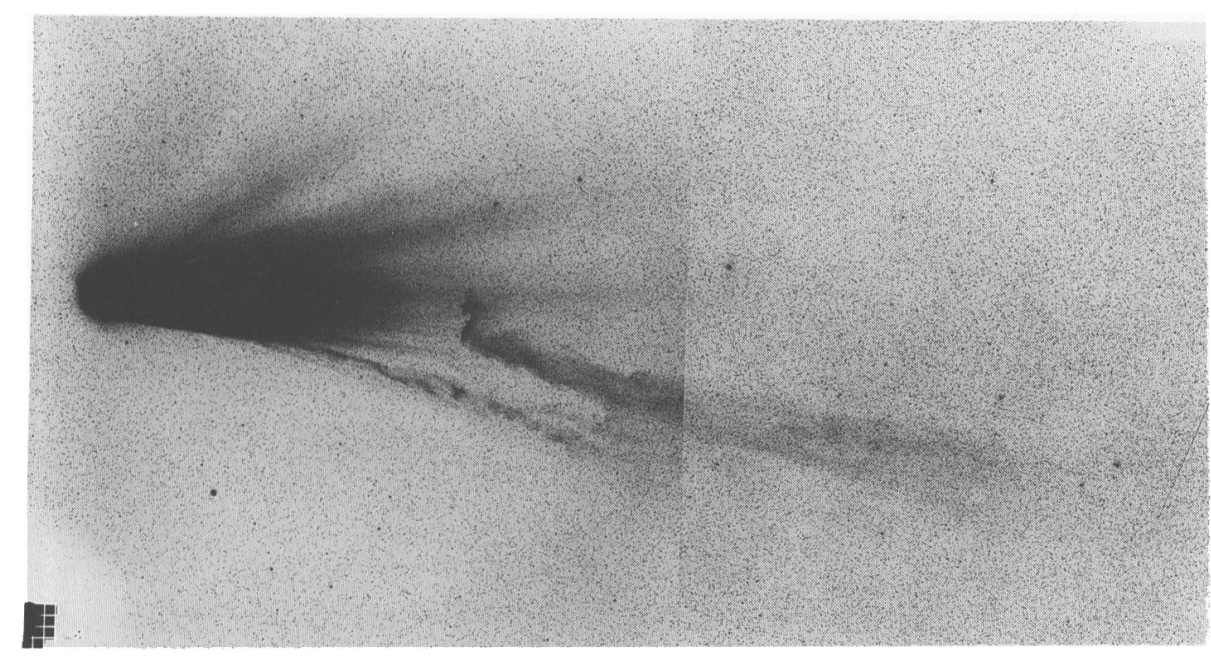

Plate 2: This photograph shows Comet Halley on 10 March 1986. It is made from two original photographs of adjacent areas of the sky to enable the complete tail to be seen.

This photograph is provided together with two films showing Halley's Comet on consecutive days, March 9 and 10, 1986, and taken exactly 24 hours apart. The coordinates of the comet and the Sun and also the distances of the comet from the Sun and from the Earth on the two dates are given. The photographs show a discontinuity in the plasma tail where the tail or part of it has broken away from the head of the comet and is separating from it. The actual break or "disconnection event" is known to have occurred on March 8.

Exercise 1. Measure the length of the plasma tail on the composite photograph and calculate the true length in Astronomical Units.

From the coordinates given (see the notes) for March 10, mark the positions of the Sun and the comet on a globe and find the angle between them. 
Measure the length of the tail on the composite print with a millimeter ruler. Measure from the sharp front of the comet's head; the end of the tail, which is faint, is not so easy to estimate. Find the scale of the print by comparing the distances apart of some bright star images on the print and on the film which has a scale of 1.12 arcminutes per millimeter. Convert the length of the tail to degrees.

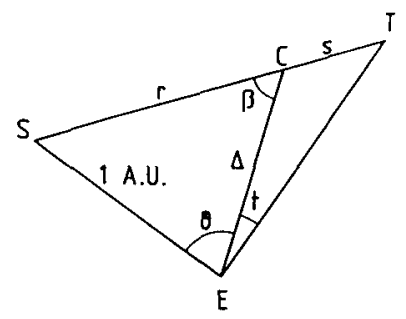

Figure 2

The diagram (Figure 2) shows the configuration of Earth, Sun, comet, and tail. The distance of the Earth from the Sun is 1 Astronomical Unit (A.U.); the distance of the comet from the Sun is $r$ A.U. and of the comet from the Earth $\delta$ A.U. The length of the tail is $s$ A.U. The angular distance between the comet and the Sun as seen from Earth is $\theta$; the angular length of the tail is $t$.

Solution by trigonometry: Solve the triangle SCE to obtain the angle $\beta$. In the small triangle CET the angle at $\mathrm{T}$ is $(\beta-t)$; the sine formula in this small triangle gives

$$
s / \sin t=\delta / \sin (\beta-t)
$$

Substitute

$$
\begin{aligned}
& \delta=1.05 \text { A.U. (given) } \\
& \theta=49^{\circ} \text { (measured on globe) } \\
& t=10^{\circ} \text { (measured on photograph) } \\
& \beta=62^{\circ} \text { (from triangle) }
\end{aligned}
$$

The result is $s=0.23 \mathrm{~A}$.U.

Solution by construction: The solution may also be found without the use of trigonometry. On millimeter graph paper draw the triangles to scale, starting with the line SE and using a suitable scale (e.g., $10 \mathrm{cms}$ to 1 A.U.). Mark the angle $\theta$ with a protractor; draw a line along this direction and mark off the position of $\mathrm{C}$ to scale. Mark also the angle $t$ and the direction to $\mathrm{T}$. Join $\mathrm{S}$ to $\mathrm{C}$ and continue until the line crosses the other lines at $\mathrm{C}$ and $\mathrm{T}$. Measure the distance $s$ with a millimeter ruler to give the length of the tail on the scale of the diagram.

Exercise 2. Calculate the average velocity of the disconnected tail relative to 
the comet in meters per second, assuming that the motion is in the direction of the tail.

Measure the distance of the disconnection from the head of the comet using a millimeter ruler on each of the films and convert these distances to degrees. The subsequent calculation is the same as in Exercise 1, replacing $t$ in the diagram by the difference between the first and second positions of the disconnection. The angle $\theta$ in this case is the angle between the Sun and the comet plus the angle between the comet and the position of the disconnection on March 9. The distance moved should be given to decimals of a degree.

Angle $\beta$ (from solution of triangle or from construction) $=60^{\circ}$

Angular distance moved by the disconnection between March 9 and March 10 $(t)=102 \mathrm{~mm}$ on film $=1.9^{\circ}$

$\Delta$ (mean for March 9 and 10 , given $)=1.06$ A.U.

Linear distance moved $(s)=\Delta \sin 1.9^{\circ} / \sin 58^{\circ}=0.041 \mathrm{~A} . \mathrm{U}$.

Interval between the two photographs $=24$ hours; 1 A.U. $=1.5 \times 10^{11}$ meters. Therefore rate of motion of disconnection $=0.041 \times 1.5 \times 10^{11} / 24 \times 3600=7.1 \times$ $10^{4}$ meters per second.

\section{Appendix 3: Globular Cluster}

Globular clusters, which may contain 100,000 to a million stars, have very high densities of stars in their central regions; their centers are therefore quite unresolved on photographs. Globular clusters are held together by their own gravity, which is able to withstand the disrupting gravitational influence of the Galaxy as a whole. An estimate of the total mass of a globular cluster may be made by assuming that the cluster is tidally limited by the Galaxy, that is, that the differential gravitational force between the extremities of the cluster exercised by the Galaxy is balanced by the self gravitational force of the cluster itself. The effect of the differential gravitational force is similar to the tides on the Earth's oceans caused at opposite sides of the globe by the Moon.

Let $r$ be the distance of the cluster from the center of the Galaxy, looked upon as a point where the mass of the Galaxy interior to the cluster is situated. Let $R$ be the radius of the cluster. One side of the cluster is nearer to the Galaxy and the opposite side more distant from it by an amount $\mathrm{R}$. This differential or tidal force is not capable of disrupting the cluster because the cluster has sufficient mass to hold itself together. The balance depends on the ratio of the masses of the cluster and the Galaxy, and the ratio of the cluster's radius $\mathrm{R}$ to its distance from the center of the Galaxy $r$. The formula connecting these ratios, assuming that the cluster is in a circular orbit around the center of the Galaxy, is:

$$
m / \mathrm{M}=3.5(\mathrm{R} / r)^{3}
$$




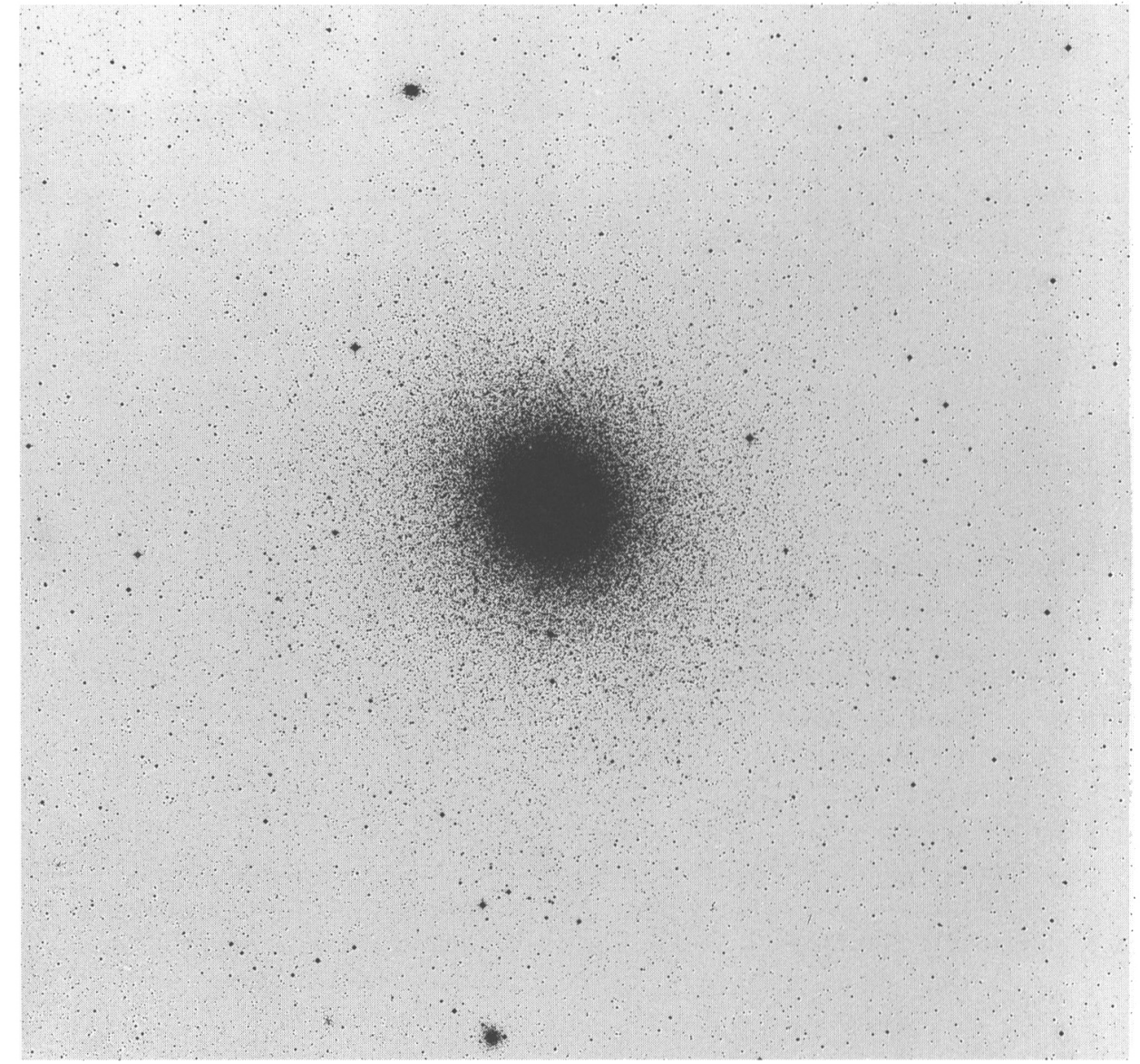

Plate 3: The large globular cluster 47 Tucanae (NGC 104) as it appears on one of the films. The small clusters seen belong to the Small Magellanic Cloud, which also appears on the film.

Exercise 1. Find the radius of the globular cluster from radial star counts. The number of stars per unit area is to be plotted as a function of distance from the center of the cluster. It is unrealistic to attempt to count all the stars; counts in one (or more) radial directions should be made, using a transparent grid laid over the film on the light box (Figure 3a). A grid with $1 \mathrm{~mm}$ squares is very suitable and viewing should be done with a x10 hand magnifier.

Choose a diameter of the cluster which is free of large star images which might interfere with the counting. Lay the grid very carefully so that the row of squares to be counted goes through the very center of the black core of the cluster. Attach the grid firmly to the protective envelope of the film. Count and note down the number of stars per square and distance of each square from the center, continuing the counts until well beyond the obvious termination of the cluster. 


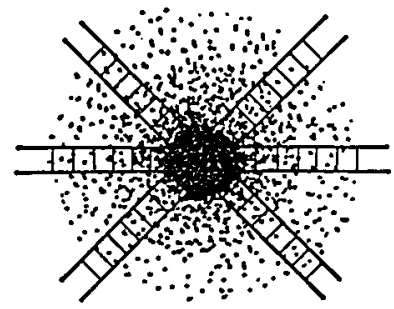

Figure 3a

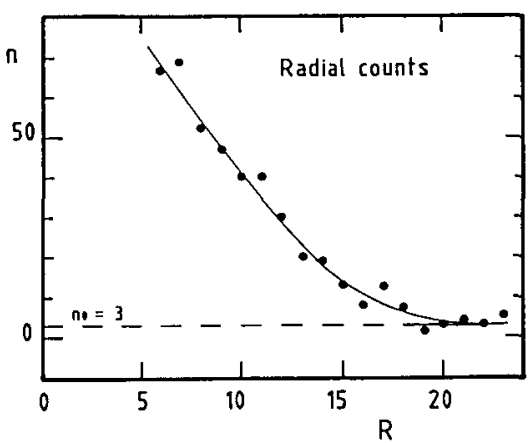

Figure $3 b$

Many very faint stars will be seen. It is not necessary to include the faintest ones, but it is important to count to the same limit of brightness throughout. As the center of the cluster is approached the stars become very numerous, with many overlapping and blended images. However, these regions need not be counted; the purpose is to find the outer limit of the cluster.

Plot the numbers as a function of the distance from the center. The limit of the cluster is the point at which the numbers level off to a constant number, representing the foreground and background stars in the general field. This limit is not always easy to assess because of statistical fluctuations in the numbers of field objects. The cluster may not be perfectly symmetrical, and the field objects not uniform; therefore the more sets of counts made, the better the estimated result.

Figure $3 \mathrm{~b}$ show one sample radial scan. The background count is 3 per unit area and the limit is estimated at $22 \mathrm{~mm}$ on the original film.

The distance of the cluster (given) is 4.1 kiloparsec. The radius is $22 \times 1.12$ arcminutes. Convert to radians and multiply by 4.1 to give the radius in linear measure. ( 1 radian $=2.06 \times 10^{5}$ arcseconds). The result is 0.029 kiloparsecs or 29 parsecs.

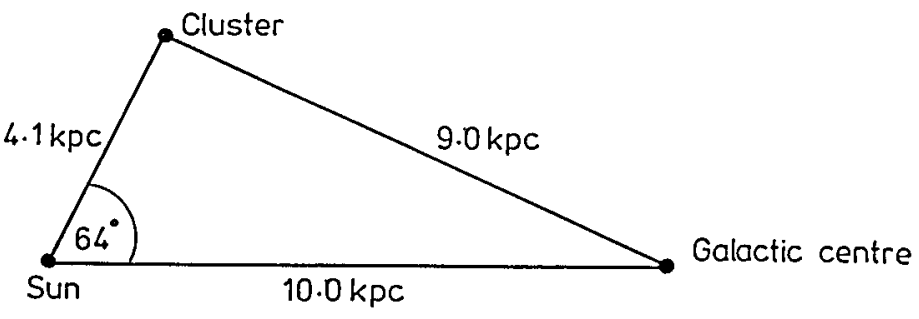

Figure 3c

Exercise 2. Calculate the mass of the cluster from the tidal radius and other data.

The coordinates of the cluster (in galactic coordinates) and the distance of the Sun from the galactic center are given. 
From the coordinates the angular distance $\theta$ between the cluster and the galactic center (coordinates 0,0 ) are found either by trigonometry or by using a globe. In the triangle in the diagram, the distance $r$ of the cluster from the galactic center is found by trigonometry or by drawing the triangle to scale (Figure 3c).

Coordinates of the cluster $\left(306^{\circ},-45^{\circ}\right)$. Angle $\theta=64^{\circ}$ (from globe or by trigonometry). Distance to galactic center $=10$ kiloparsecs (given). Distance to cluster $=4.1$ kiloparsecs (given). Distance of cluster from galactic center $(r)=9.0$ kiloparsecs (from triangle). Radius of cluster $(R)=29 \times 10^{-3}$ kiloparsecs (exercise 1). Mass of Galaxy $(M)=2 \times 10^{11}$ solar masses (given).

$$
\begin{aligned}
& m / \mathrm{M}=3.5 \times\left(29 \times 10^{-3} / 9.0\right)^{3}=1 \times 10^{-7} \\
& m=2 \times 10^{4} \text { solar masses }
\end{aligned}
$$

Accuracy of the result. The value of the tidal radius obtained from star counts probably tends to be underestimated because in the outer zones a very low level of counts is not distinguishable above the background ("noise") level. The distance to the center of the Galaxy and the mass of the Galaxy are quantities which are not yet agreed on with certainty.

\section{Appendix 4: Spectral Classification}

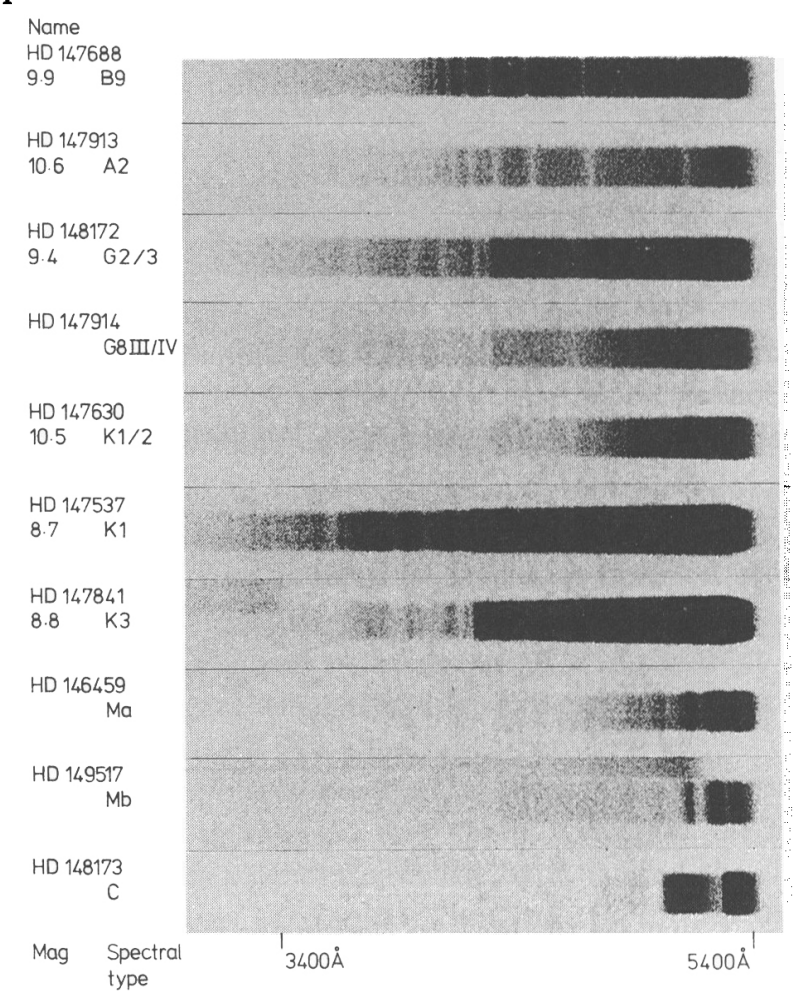

Plate 4: Examples of standard stars of different spectral types as seen on the objective prism film copies. 


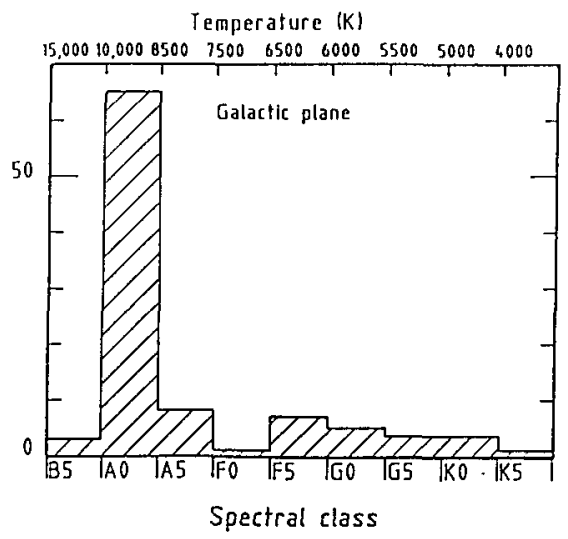

Figure $4 \mathrm{a}$

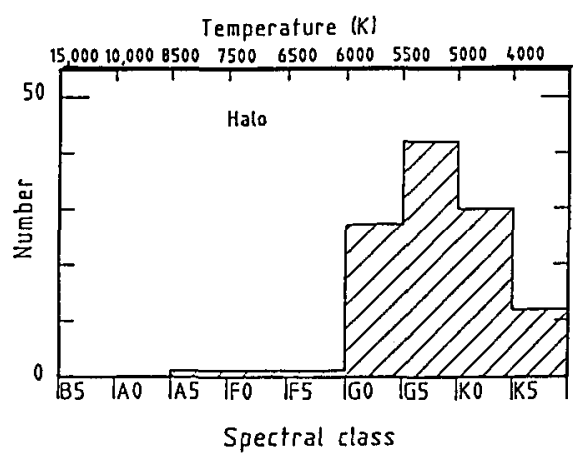

Figure $4 \mathrm{~b}$

With a little practice it is easy to classify stars to one spectral class (or better). The exercise is to classify stars in similarly sized small areas of one photograph taken in the plane of the galaxy and of another taken perpendicular to the plane. The difference in stellar populations between the disk and arms of the galaxy (which generally contain young, hot stars) and the halo (which contains older stars) is easily seen in the histograms (Figures $4 \mathrm{a}$ and $4 \mathrm{~b}$ ).

\section{Appendix 5: Cluster of Galaxies}

There are some hundreds of galaxies in the field, made up of the cluster members and also the individual lone galaxies which are scattered more or less uniformly over the sky. The student's task is to decide, by careful analysis of counts over the whole film, where the center of the group lies and to find the average density of background galaxies. At high magnification it is possible to classify the galaxies according to Hubble's "tuning fork" scheme. The classification, together with the apparent diameter of each galaxy, gives an estimate of its distance. The distance of this cluster, inferred from the redshift of its members, is $\mathbf{4 5}$ megaparsecs. The spatial distribution of galaxies in the cluster is found by counting and fitting to models; from this the average separation of galaxies is found $(\sim 3 \mathrm{Mpc})$. This information combined with the internal velocity dispersion, known from spectroscopy, gives the cluster's total mass ( $7.5 \times 10^{14}$ solar masses). 


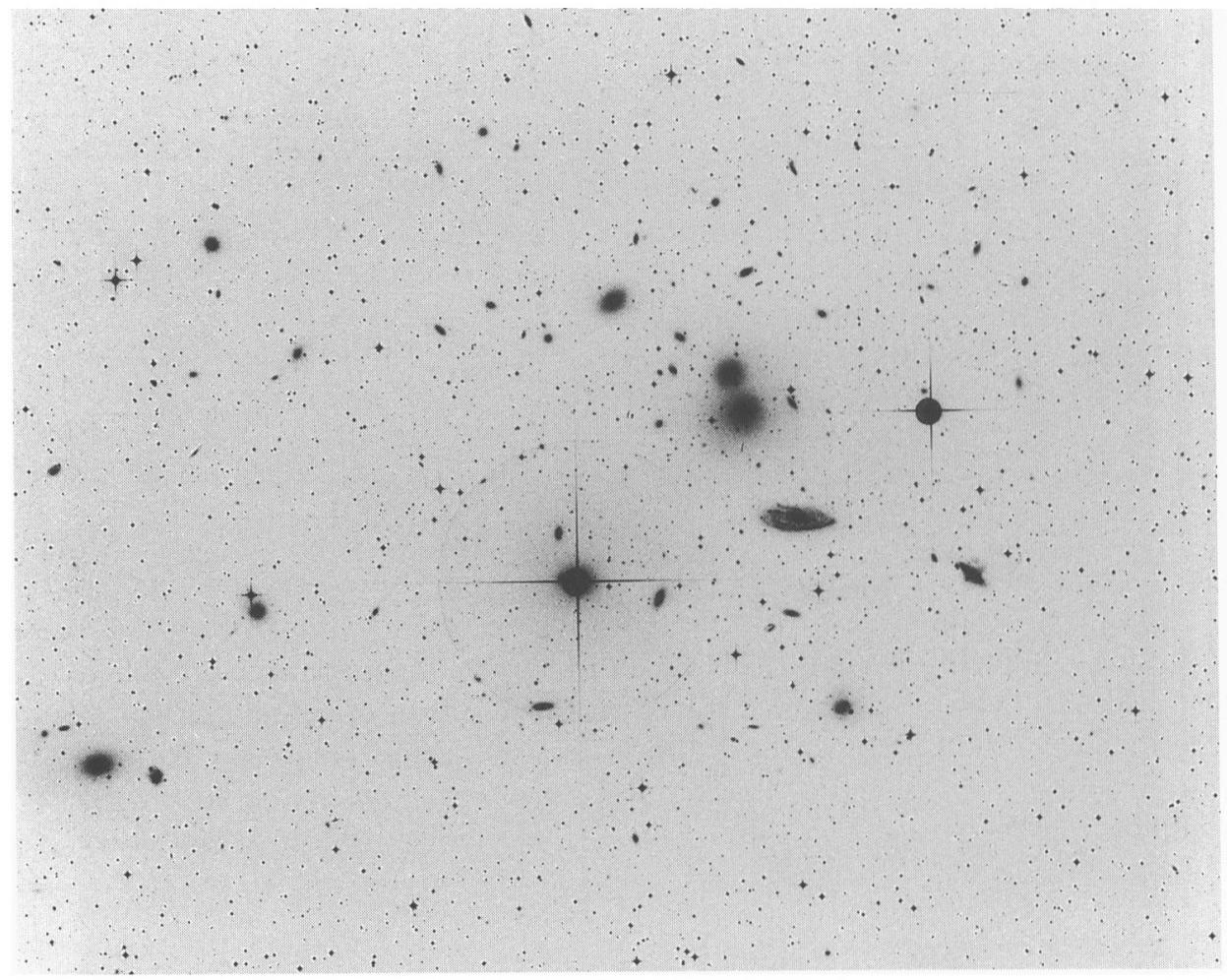

Plate 5: Abell 1060. The photograph shows only the central part of the cluster and covers only a small part of the provided film.

\section{CLASSIFICATION OF STELLAR SPECTRA}

\section{M.M. Dworetsky}

University of London Observatory, University College London, Mill Hill Park, London NW7 2QS, U.K.

Summary: The objectives of this exercise are to gain familiarity with the appearance of the spectra of stars, to understand some of the temperature and luminosity criteria used to classify spectra, and to gain insights into the relationship between the appearance of stellar spectra and physical conditions in stellar atmospheres.

Our experience with exercises in which beginning undergraduate astronomy degree students are challenged to classify "real" stellar spectra according to Harvard 